\section{G377 NEW MODELS OF CARE FOR CHILDREN: TESTING INTEGRATING PRIMARY AND SECONDARY CARE CLINICS}

${ }^{1} \mathrm{R}$ Goodwin, ${ }^{1} \mathrm{C}$ Lemer, ${ }^{2} \mathrm{R}$ Satherley, 1,2, Wolfe. ${ }^{1}$ Children and Young People's Health Partnership, Evelina London Children's Hospital, London, UK; ${ }^{2}$ Health and Social Care Research, King's College London, London, UK

\subsection{6/archdischild-2018-rcpch.366}

Aims Integrated General Practitioner-Paediatrician 'In-Reach' clinics aim to improve access to care and professional learning across primary-secondary care. The aim of this pilot project is to understand the range of presenting conditions and outcomes among children referred by General Practitioners (GP) to GPs-Paediatrician 'In-Reach' clinics, instead of hospital outpatients'.

Methods Retrospective case review of 101 patient's notes. Cases identified through an electronic search of consultations during a 6 month period in 2017. 202 cases were identified, listed alphabetically by patient surname, and alternate records were chosen systematically for inclusion. A senior clinician identified the presenting problem using ICD10 codes grouped into tier one categories, and clinic outcomes.

Results Our sample comprised 101 children (0-18 years) presenting with 115 problems. Constipation, eczema, and asthma accounted for $25 \%$ of problems. The top 5 ICD10 categories, representing $60 \%$ of the total, were digestive, dermatological, musculoskeletal, nervous, and respiratory problems ('other' comprised a large range of conditions each with small numbers of patients) (table 1).

\begin{tabular}{ll}
$\begin{array}{l}\text { Abstract G377 Table } 1 \\
\text { referred to In-Reach clinics }\end{array}$ & Presenting problems among children \\
\hline ICD10 categories & Percentage of total referrals \\
\hline Digestive & 18 \\
Skin/subcutaneous tissue & 16 \\
Muscloskeletal & 10 \\
Respiratory & 8 \\
Nervous & 8 \\
Other & 40
\end{tabular}

Nearly a third of problems required only reassurance, one in five required a prescription, and a specialist referral was made for $24 \%$ of children seen (table 2).

\begin{tabular}{ll}
$\begin{array}{l}\text { Abstract G377 Table 2 } \\
\text { clinics }\end{array}$ & Outcome for children seen in in-reach \\
\hline Management plan recommendation & Outcome (percentage of total) \\
\hline Reassurance & 28 \\
Specialist hospital referral \pm medication & 24 \\
Medication recommended with GP management & 22 \\
Self-management & 8 \\
Primary care referral \pm medication & 8 \\
Continue GP plan & 6 \\
GP monitoring & 4
\end{tabular}

Conclusion A substantial proportion of children had common problems requiring reassurance or simple management in primary care. Specialist referral was required for $24 \%$ of children. If this sample is representative, approximately three quarters of children, who would otherwise have been referred for hospital outpatients' appointments, could be seen and managed with support in primary care settings. These preliminary results suggest that integrating care across the primarysecondary care interface could help improve access to care.

\section{G378 AUGMENTING THE SAFETY NETTING PROCESS \& REDUCING UNNECESSARY RE-PRESENTATIONS - A QUALITY IMPROVEMENT PROJECT}

${ }^{1,2,3} \mathrm{~S} J$ Gray, ${ }^{1} \mathrm{~K}$ Pryde, ${ }^{1} \mathrm{~S}$ Patel. ${ }^{1}$ Paediatrics, Southampton Childrens Hospital, Southampton,
UK; ${ }^{2}$ Quality Improvement, Health Education England (Wessex), Winchester, UK;
${ }^{3}$ Department of Medicine, Imperial College, London, UK

10.1136/archdischild-2018-rcpch.367

Background Despite the reducing incidence of life-threatening illness in children, health-seeking behaviour and demand on secondary services, such as Paediatric Assessment Units (PAU's), has increased. Re-presentations via temporary open access anecdotally represent a significant proportion of the workload and may be avoidable with improved safety netting processes.

\section{Aims}

To identify the scale of unnecessary re-presentations to PAU's across our region and seek views from health care professionals (HCP's), parents and carers to guide improvements to the process.

To implement changes to reduce unnecessary re-presentations as guided by the scoping work.

Methods Scoping work across the region assessing facilities, procedures, re-presentation rates and length of stay was performed. Views on safety netting from health care professionals and parents and carers were acquired via purpose-designed questionnaires. Using QI methodology and a series of PlanDo-Study-Act (PDSA) cycles, a document of all safety netting resources was introduced in the pilot centre. The primary outcome measured was proportion of children seen on PAU returning via temporary open access. Secondary outcomes measures included documentation of safety net advice, proportion staying for less than 4 hours and the proportion physically in receipt of an information leaflet.

Results Continuous data plotted on run charts showed a significant reduction in the proportion of children seen returning via temporary open access from $11.5 \%$ to $4.2 \%$ following introduction and promoting of the safety netting resource. There was no significant change in the documentation rates or proportion staying less than 4 hours but $100 \%$ of parents physically received an information leaflet in the post-intervention period.

Conclusion The results demonstrate that streamlining and simplifying processes for HCP's in giving safety net advice and information reduced re-presentations. More powerfully, breaking down hierarchy and empowering staff across all levels to share ownership of the safety netting process led to a culture change across PAU. Further work is required to improve on documentation processes and delineate optimal safety netting strategy. The Child Health Information and Leaflet Directory (CHILD; http://www.piernetwork.org/child.html) has been developed to implement this work across the region transcending primary and secondary care. 